\title{
Study on Chemical Constituents and Bioactivities from Eucalyptus globulus
}

\author{
Shudan Zheng, ${ }^{a}$ Hui Fang, ${ }^{a}$ and Jifeng Liu* ${ }^{\star, a, b}$ \\ ${ }^{a}$ School of Pharmaceutical Sciences, Institute of Drug Discovery and Development, Key Laboratory of \\ Advanced Pharmaceutical Technology, Ministry of Education of China, Zhengzhou University, \\ Zhengzhou, Henan 450001, China \\ ${ }^{b}$ Collaborative Innovation Center of New Drug Research and Safety Evaluation, Zhengzhou, \\ Henan 450001, China \\ Email: liujf2009y@126.com (J. L.)
}

\begin{abstract}
Eucalyptus globulus (E. globulus) is a tall timber tree of genus Eucalyptus (Myrtaceae), and it is mainly distributed in the southern and southwestern China. The dry fruits of E. globulus, called as 'Yi-Kou-Zhong' in Chinese, have been commonly used as a traditional Chinese medicine to treat in flammation, eczema, and influenza. Herein, we summarized the chemical constituents and bioactivities of the Eucalyptus globulus.
\end{abstract}

Keywords Eucalyptus globulus, chemical constituents, bioactivities

\section{Introduction}

Eucalyptus globulus is one of the most widely planted Eucalyptuses in the world. E. globulus is native to Tasmania and Australia. It was recorded that this plant was firstly introduced to China in late 1890s. ${ }^{[1]}$ Nowadays, it is widely cultivated in southern and southwestern China, especially in Yunnan and Jiangxi provinces. It is commonly known as button seven, red trumpet flowers, victory fruit, and so on. ${ }^{[2]}$ The chemical composition in E. globulus can be divided into volatile oil, triterpenoids, monoterpenes, sesquiterpenes, phloroglucinol derivatives, flavonoids, tannins. It is reported that this plant has anti-tumor, anti-inflammatory, anti-AIDS, anti-bacterial, anti-virus and other functions. ${ }^{[3]}$

Herein, we utilized "chemical constituents of Eucalyptus globulus" and 'activities of "Eucalyptus globulus" as keywords, and relevant literatures and books were searched on SciFinder, Web of Science, China National Knowledge Infrastructure academic database and website. Herein, we summarized the chemical constituents and activities of E. globulus.

\section{Chemical Constituents}

Up to date, about 123 compounds have been isolated from E. globulus, including terpenes, flavonoids, organic acids, sesquiterpene, tannins, triterpene and phloroglucinol derivatives. $^{[3]}$

\section{Monoterpenes and sesquiterpenes}

Five monoterpenes and sesquiterpenes have been isolated from E. globulus, which were identified as camphor (1), camphene (2), oleuropeic acid (3), elemol (4), globulol (5). ${ }^{[4-6]}$ Their structures are shown in Figure 1.

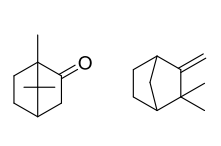

1<smiles>CC(C)(O)C1CC=C(C(=O)O)CC1</smiles>

3

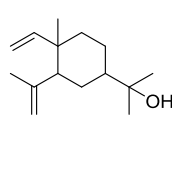

4

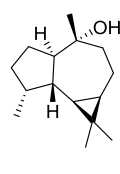

5
Figure 1 Chemical structures of monoterpenes and sesquiterpenes isolated from $E$. globulus.

\section{Triterpenoids}

13 compounds were isolated from the fruits of $E$. globulus, named as $3 \beta$-acetoxyurs-11-en-28,13-olide (6), betulonic acid

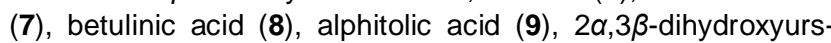
12-en-28-oic acid (10), ursolic acid (11), 11,12-dehydrousolic acid lactone (12), euscaphic acid (13), 3-oxo-ursolicacid (14), corosolic acid (15), 3 $\beta, 13 \beta$-dihydroxy-urs-11-en-28-oic acid-13lactone (16), llelatifol D (17), colosolic acid (18). ${ }^{[2,7-8]}$ Above-mentioned compounds 14 and 18 were isolated from the fruits of $E$. globulus for the first time. 16 compounds were isolated from the stem bark of $E$. globulus, named as $\beta$-amyrin (19), acetylursolic acid (20), acetyloleanolic acid (21), acetylbetulinic acid (22), erythrodiol (23), uvaol (24), $11 \alpha$-methoxyacetylursolic acid methyl ester (25), betulinic acid methyl ester (26), cis-p-methoxy-cinnamoyloxyursolic acid methyl ester (27), cis-p-methoxy-cinnamoyl-oxyoleanolic acid methyl ester (28), ursolic acid methyl ester (29), methyl 33,23-diacetoxy-12-ursen-28-oate (30), oleanolic acids (31),

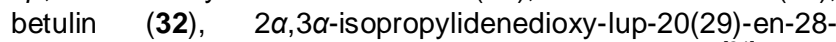
oieaeid (33), $3 \beta$-formyloxyurs-11-en-28,13-olide (34). ${ }^{[24]}$ Their structures are shown in Figure 2.

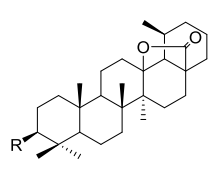

6: $\mathrm{R}=\mathrm{CH}_{3} \mathrm{COO}$ 12: $\mathrm{R}=\mathrm{OH}$

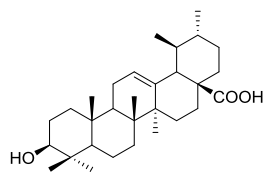

11

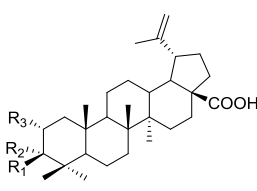

$7: \mathrm{R}_{1}+\mathrm{R}_{2}=\mathrm{O}, \mathrm{R}_{3}=\mathrm{H}$ 8: $\mathrm{R}_{1}=\mathrm{OH}, \mathrm{R}_{2}=\mathrm{H}, \mathrm{R}_{3}=\mathrm{H}$ 9: $\mathrm{R}_{1}=\mathrm{OH}, \mathrm{R}_{2}=\mathrm{H}, \mathrm{R}_{3}=\mathrm{OH}$

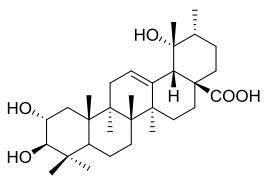

13

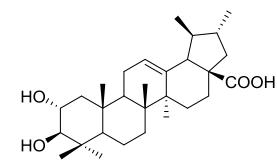

10

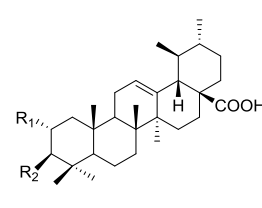
$15: \mathrm{R}_{1}=\mathrm{OH}, \mathrm{R}_{2}=\mathrm{OH}$
14: $\mathrm{R}_{1}=\mathrm{H}, \mathrm{R}_{2}=\mathrm{O}$ 


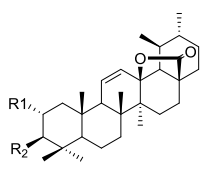

$16: \mathrm{R}_{1}=\mathrm{H}, \mathrm{R}_{2}=\mathrm{OH}$

$17: \mathrm{R}_{1}=\mathrm{OH}, \mathrm{R}_{2}=\mathrm{OH}$

$34: \mathrm{R}_{1}=\mathrm{H}, \mathrm{R}_{2}=\mathrm{OCHO}$

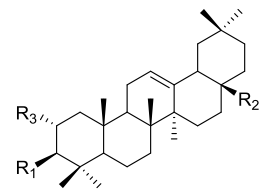

19: $\mathrm{R}_{1}=\mathrm{OH}, \mathrm{R}_{2}=\mathrm{CH}_{3}, \mathrm{R}_{3}=\mathrm{H}$

21: $\mathrm{R}_{1}=\mathrm{OAc}, \mathrm{R}_{2}=\mathrm{COOCH}_{3}, \mathrm{R}_{3}=\mathrm{H}$

23: $\mathrm{R}_{1}=\mathrm{OH}, \mathrm{R}_{2}=\mathrm{CH}_{2} \mathrm{OH}, \mathrm{R}_{3}=\mathrm{H}$

28: $\mathrm{R}_{1}=\mathrm{P}-\mathrm{CH}_{3} \mathrm{O}-\mathrm{C}_{6} \mathrm{H}_{4}-\mathrm{CH}=\mathrm{CH}-$

$\mathrm{COO}, \mathrm{R}_{2}=\mathrm{COOCH}_{3}, \mathrm{R}_{3}=\mathrm{H}$

$31: \mathrm{R}_{1}=\mathrm{OH}, \mathrm{R}_{2}=\mathrm{COOH}, \mathrm{R}_{3}=\mathrm{OH}$

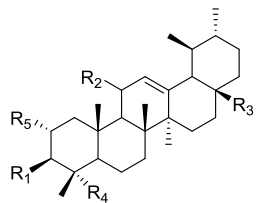

$20: \mathrm{R}_{1}=\mathrm{OAc}, \mathrm{R}_{2}=\mathrm{H}, \mathrm{R}_{3}=\mathrm{COOCH}_{3}, \mathrm{R}_{4}=\mathrm{CH}_{3}, \mathrm{R}_{5}=\mathrm{H}$

24: $\mathrm{R}_{1}=\mathrm{OH}, \mathrm{R}_{2}=\mathrm{H}, \mathrm{R}_{3}=\mathrm{CH}_{2} \mathrm{OH}, \mathrm{R}_{4}=\mathrm{CH}_{3}, \mathrm{R}_{5}=\mathrm{H}$

25: $\mathrm{R}_{1}=\mathrm{OAc}, \mathrm{R}_{2}=\mathrm{CH}_{3} \mathrm{O}, \mathrm{R}_{3}=\mathrm{COOCH}_{3}, \mathrm{R}_{4}=\mathrm{CH}_{3}, \mathrm{R}_{5}=\mathrm{H}$

27: $\mathrm{R}_{1}=\mathrm{P}-\mathrm{CH}_{3} \mathrm{O}-\mathrm{C}_{6} \mathrm{H}_{4}-\mathrm{CH}=\mathrm{CH}-\mathrm{COO}, \mathrm{R}_{2}=\mathrm{H}, \mathrm{R}_{3}=\mathrm{COOCH}_{3}, \mathrm{R}_{4}=\mathrm{CH}_{3}, \mathrm{R}_{5}=\mathrm{H}$

29: $\mathrm{R}_{1}=\mathrm{OH}, \mathrm{R}_{2}=\mathrm{H}, \mathrm{R}_{3}=\mathrm{COOCH}_{3}, \mathrm{R}_{4}=\mathrm{CH}_{3}, \mathrm{R}_{5}=\mathrm{H}$

30: $\mathrm{R}_{1}=\mathrm{OAc}, \mathrm{R}_{2}=\mathrm{H}, \mathrm{R}_{3}=\mathrm{COOCH}_{3}, \mathrm{R}_{4}=\mathrm{CH}_{2} \mathrm{OAc}, \mathrm{R}_{5}=\mathrm{H}$

Figure 2 Chemical structures of triterpenoids isolated from $E$. globulus.

\section{Phloroglucinol derivatives}

Phloroglucinol derivatives are the main compounds of $E$. globulus. Tian et al. ${ }^{[9,10]}$ obtained five new phloroglucinol glyoroglu-cinol, named as eucalmainosides A (35), eucalmainosides B (36), eucal-mainosides C (37), eucalmainosides $D(\mathbf{3 8})$, eucalmainosides $E(\mathbf{3 9})$, and a carbon glycoside, named 8- $\beta$-C-glucopyranosyl-5,7-dihydroxy-2isobutylchromone (40). Eight compounds were isolated from $E$. globulus, which were identified as eucalyptal A (41), eucalyptal B (42), eucalyptal C (43), ${ }^{[11]}$ eucalyptals D (44), eucalyptals $E(45),{ }^{[12]}$ eucalyptin A (46), ${ }^{[13]}$ euglobal R1 (47), euglobal R2 (48). ${ }^{[23]}$ Qin et al. ${ }^{[14]}$ obtained ten new formyl-phloroglucinol-terpene meroterpenoids, named eucalypglobulusals A-J (49-58). Pham et al. ${ }^{[15]}$ obtained five new phloroglucinol derivatives, named as eucalyptins $E-G(59-61)$, and thirteen known compounds were also isolated from the fruits of $E$. globulus, named as eucalyptin B (62), macrocarpal A (63), macrocarpal B (64), macrocarpal C (65), macrocarpal D (66), macrocarpal E (67), macrocarpal Q (68), eucarobustol E (69), euglobal-V (70), euglobal-III (71), 1-(2,6-dihydroxy-4methoxy-3,5-dimethylphenyl)-2-methylbutan-1-one (72), 1-(2,4dihydroxy-6-methoxy-3,5-dimethylphenyl)-3-meth-ylbutan-1one (73), cypellocarpa $C(74)$. Seven phloroglucinol derivatives were accessed from the leaves of $E$. globulus, named as eucalyptone (75), macrocarpal $H$ (76), macrocarpal I (77), macrocarpal $\mathrm{J}$ (78), euglobal-IVb (79), euglobal-VII (80), euglobal-IX (81). Two compounds were isolated from the stem bark of $E$. globulus, named eucalyptone $G(82)$, rhodomyrtone (83). ${ }^{[23,30]}$ Their structures are shown in Figure 3.

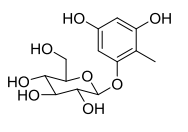

35

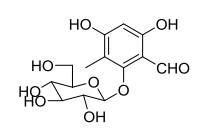

38

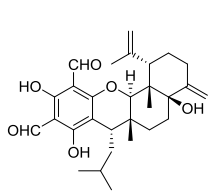

41

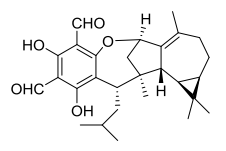

44
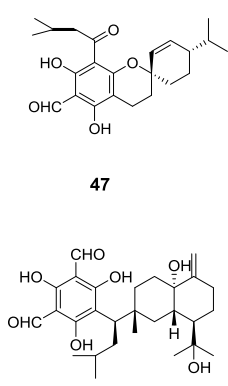

50

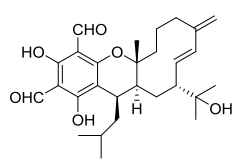

(1)

57

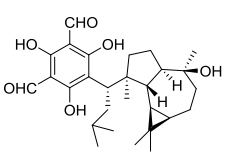

60

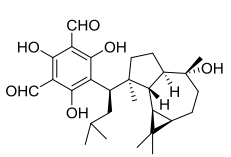

63

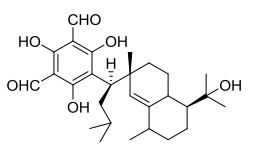

66

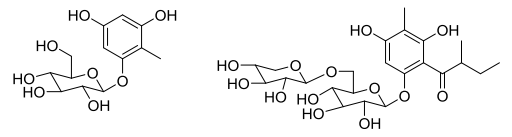

36

37
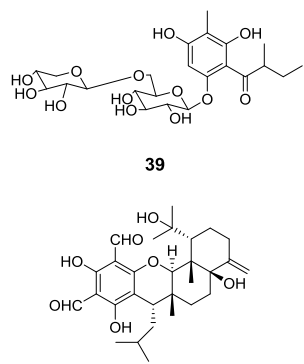

42
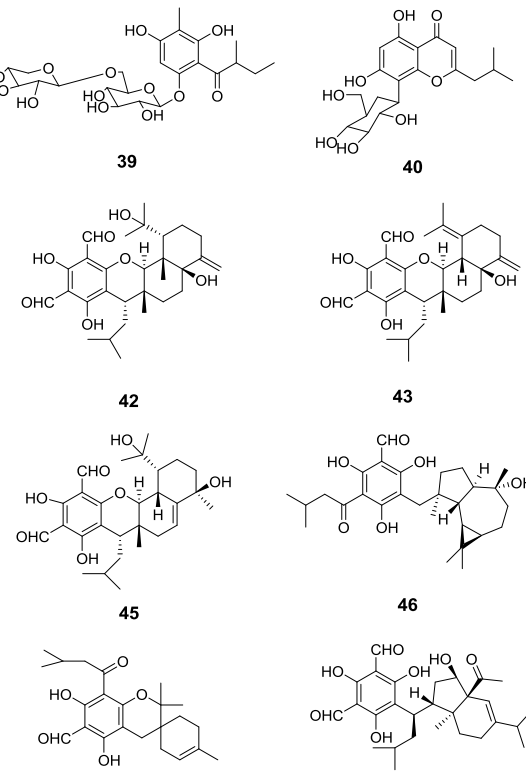

43
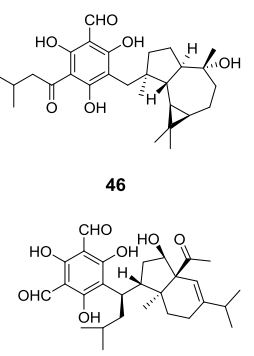

49
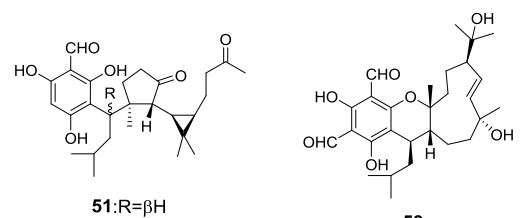

$52: R=\alpha H$
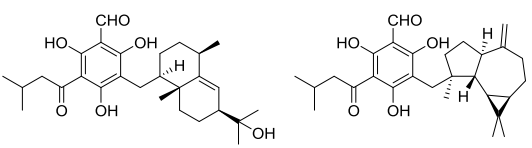

56

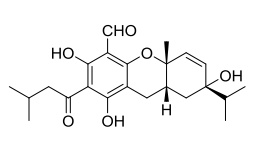

58

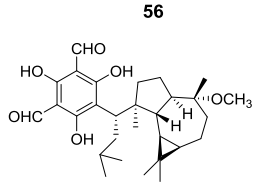

59
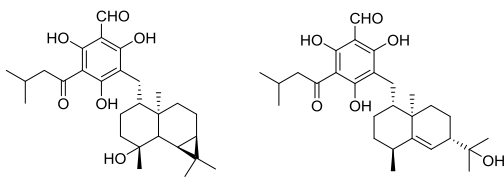

61
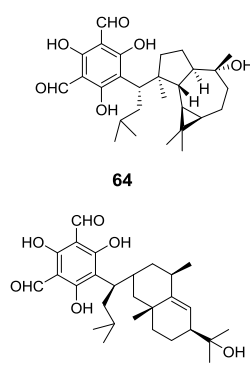

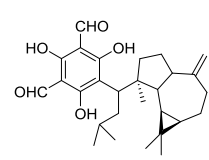

65

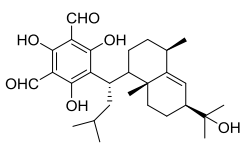

68 
<smiles>CCCC(c1c(O)c(C=O)c(O)c(C=O)c1O)[C@@H]1CCC2C(C)CCC1C2(C)C</smiles>

69<smiles>CCC(C)C(=O)c1c(O)c(C)c(OC)c(C)c1O</smiles>

72<smiles>CC(=O)CCC(C(C)(C)C)C1(C)C(=O)CCC(c2c(O)c(CO)c(O)c(C=O)c2C=O)C1C</smiles>

75

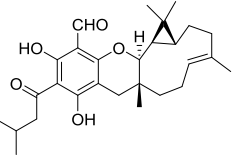

80<smiles>CCCC1c2c(cc(O)c(C(=O)CC(C)C)c2O)OC2C1C(=O)C(C)(C)C(=O)C2(C)C</smiles>

Figure 3 Chemical structures of phloroglucinol derivatives isolated from $E$. globulus.

\section{Flavonoids}

Flavonoids are one of the earlier developed components in E. globulus. Sixteen flavonoids were isolated from thefruits of $E$. globulus, which were identified as 5-hydroxyl-4',7-dimethoxy6-methyflavone (84), 8- $\beta$-C-glucosyl-5,7-dihydroxy-2-methylchro-manone (85), 6- $\beta$-C-glucosyl-5,7-dihydroxy-2-methylchromanone (86), 8- $\beta$-C-glucopyranosyl-5,7-dihydroxy-2isobutylchromone (87), 8- $\beta$-C-glucopyrano-syl-5,7-dihydroxy-2isopropylchromone (88), quercetin $3-O-\beta-D$-glucopyranoside (89), quercetin 3-O- $\beta$ - $D$-glucuronide-6"-methyl ester (90), rhamnazin (91), rhamnetin (92), eriodictyo (93), quercetin (94), taxifolin (95), engelitin (96), catechin (97), kaempferol (98), 5-hydroxyl-4', 7-dimethoxy-6,8-dimethyflavone (99). ${ }^{[16-20]}$ Seven compounds were isolated from the stem bark of $E$. globulus, which were identified as isorhamnetin-7-O- $\beta$ - $D$-glucoside (100), isorhamnetin-7-O- $\beta$ - $D$-rhamnoside (101), mearnsetin (102), mearnsetin-7-O- $\beta$ - $D$-rhamnoside (103), luteolin (104), isorhamnetin (105), naringenin (106). ${ }^{[24]}$ Their structures are shown in Figure 4.

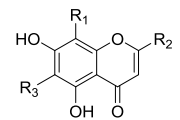

85: $\mathrm{R}_{1}=\beta$-D-glucopyranoside, $\mathrm{R}_{2}=\mathrm{CH}_{3}, \mathrm{R}_{3}=\mathrm{H}$ 86: $\mathrm{R}_{1}=\mathrm{H}, \mathrm{R}_{2}=\mathrm{CH}_{3}, \mathrm{R}_{3}=\beta$-D-glucopyranoside 87: $\mathrm{R}_{1}=\beta$-D-glucopyranoside, $\mathrm{R}_{2}=\mathrm{CH}_{2}$-CH$\left(\mathrm{CH}_{3}\right)_{2}, \mathrm{R}_{3}=\mathrm{H}$

88: $\mathrm{R}_{1}=\beta$-D-glucopyranoside, $\mathrm{CH}\left(\mathrm{CH}_{3}\right)_{2}, \mathrm{R}_{3}=\mathrm{H}$

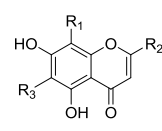

85: $\mathrm{R}_{1}=\beta$-D-glucopyranoside, $\mathrm{R}_{2}=\mathrm{CH}_{3}, \mathrm{R}_{3}=\mathrm{H}$ $86: \mathrm{R}_{1}=\mathrm{H}, \mathrm{R}_{2}=\mathrm{CH}_{3}, \mathrm{R}_{3}=\beta$-D-glucopyranoside 87: $\mathrm{R}_{1}=\beta$-D-glucopyranoside, $\mathrm{R}_{2}=\mathrm{CH}_{2}-\mathrm{CH}$ $\left(\mathrm{CH}_{3}\right)_{2}, \mathrm{R}_{3}=\mathrm{H}$

88: $\mathrm{R}_{1}=\beta$-D-glucopyranoside, $\mathrm{CH}\left(\mathrm{CH}_{3}\right)_{2}, \mathrm{R}_{3}=\mathrm{H}$
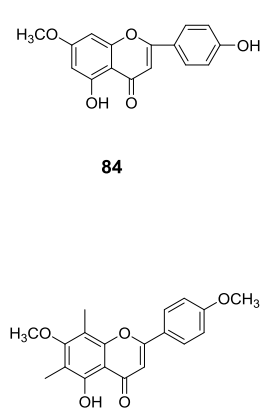

99

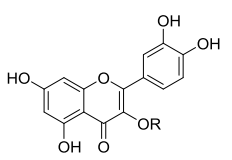

89:R= $\beta$-D-glucopyranoside $90: R=\beta$-D-glucuronide-6"-methyl ester<smiles>[R]C1=C([R])C(c2ccc(O)c([R3])c2)Oc2cc(O)cc(O)c21</smiles>

93: $\mathrm{R}_{1}=\mathrm{O}, \mathrm{R}_{2}=\mathrm{H}_{2}, \mathrm{R}_{3}=\mathrm{OH}$ 95: $\mathrm{R}_{1}=\mathrm{O}, \mathrm{R}_{2}=\mathrm{OH}, \mathrm{R}_{3}=\mathrm{OH}$ 96: $\mathrm{R}_{1}=\mathrm{O}, \mathrm{R}_{2}=\mathrm{ORha}, \mathrm{R}_{3}=\mathrm{H}$ 97: $\mathrm{R}_{1}=\mathrm{H} \mathrm{R}_{2}=\mathrm{OH} \mathrm{R}_{3}=\mathrm{OH}$<smiles>[R6]Oc1cc(O)c2c(=O)c(O)c(-c3cc([R6])c([R6])c(C)c3)oc2c1</smiles>

$100: \mathrm{R}_{1}=\mathrm{Glc}, \mathrm{R}_{2}=\mathrm{OCH}_{3}, \mathrm{R}_{3}=\mathrm{H}, \mathrm{R}_{4}=\mathrm{OH}$ 101: $\mathrm{R}_{1}=\mathrm{Rha}, \mathrm{R}_{2}=\mathrm{OCH}_{3}, \mathrm{R}_{3}=\mathrm{H}, \mathrm{R}_{4}=\mathrm{OH}$ 102: $\mathrm{R}_{1}=\mathrm{H}, \mathrm{R}_{2}=\mathrm{OH}, \mathrm{R}_{3}=\mathrm{OH}, \mathrm{R}_{4}=\mathrm{OCH}_{3}$ $103: \mathrm{R}_{1}=\mathrm{Rha}, \mathrm{R}_{2}=\mathrm{OH}, \mathrm{R}_{3}=\mathrm{OH}, \mathrm{R}_{4}=\mathrm{OCH}_{3}$ 104: $\mathrm{R}_{1}=\mathrm{H}, \mathrm{R}_{2}=\mathrm{H}, \mathrm{R}_{3}=\mathrm{OH}, \mathrm{R}_{4}=\mathrm{OH}$ 105: $\mathrm{R}_{1}=\mathrm{H}, \mathrm{R}_{2}=\mathrm{OCH}_{3}, \mathrm{R}_{3}=\mathrm{H}, \mathrm{R}_{4}=\mathrm{OH}$

Figure 4 Chemical structures of flavonoids isolated from $E$. globulus.

\section{Tannis}

Seven acid compounds were isolated from the fruits of E.globulus, which were identified as ellagic acid (107), 3-O-methylellagic acid-4'-O- $\alpha$ - $L$-rhamnopyranoside (108), valoneic acid dilactone (109), 3-O-methylellagic acid-4'-O2"-O-acetyl- $\alpha$ - $L$-rhamnopyranoside (110), 3-O-methylellgic acid (111), 3-O-methylellagic acid 4'-O- $\alpha$ - $L-2 "-O$-acetylrhamnopyranoside(112), 3-O-methylellagic acid 4'-O- $\alpha$-rhamnopyranoside (113). ${ }^{[8,17,21]}$ Ten acid compounds were isolated from the stem bark of $E$. globulus, which were identified as ellagic acids (114), 3-O-methylellagic acid-4'-rhamnoside (115), 3,4,3'-O-trimethylellagic acid (116), 3,3'-O-dimethylellagic acid (117), 3,4,3',4'-O-tetramethylellagic acid (118), 3-O-methylellagic acid 3'-O- $\alpha$-rhamno-pyranoside (119), 3-O-methylellagic acid 3'-O- $\alpha$-3"-O-acetylrhamnopyranosid (120), 3-O-methylellagic acid 3'-O- $\alpha-2 "-O$-acetylrhamnopyranoside (121), 3-O-methylellagic acid 3 '-O- $\alpha$-4"-O-acetylrhamnopyranoside (122), methyl-ellagic acid (123). ${ }^{[23]}$ Their structures are shown in Figure 5.

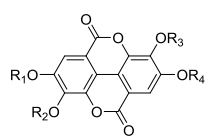

$107: \mathrm{R}_{1}=\mathrm{H}, \mathrm{R}_{2}=\mathrm{H}, \mathrm{R}_{3}=\mathrm{H}, \mathrm{R}_{4}=\mathrm{H}$

08: $R_{1}=H, R_{2}=C H 3, R_{3}=H, R_{4}=\alpha$-L-rhamnopyranoside 109: $\mathrm{R}_{1}=H, \mathrm{R}_{2}=\mathrm{H}, \mathrm{R}_{3}=\mathrm{H}, \mathrm{R}_{4}=$ Gallic acid

-D-glucopyranoside 90:R= $\beta$-D-glucuronide-6"-methyl ester

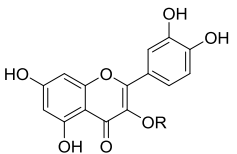

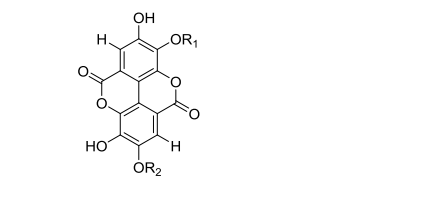

$111: \mathrm{R}_{1}=\mathrm{CH}_{3}, \mathrm{R}_{2}=\mathrm{H}$

112: $\mathrm{R}_{1}=\mathrm{CH}_{3}, \mathrm{R}_{2}=\alpha-\mathrm{L}-2$ "-O-acetylrhamnopyranoside 113: $\mathrm{R}_{1}=\mathrm{CH}_{3}, \mathrm{R}_{2}=\alpha$-L-rhamnopyranoside

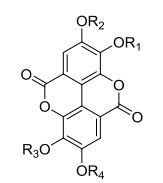

114: $\mathrm{R}_{1}=\mathrm{H}, \mathrm{R}_{2}=\mathrm{H}, \mathrm{R}_{3}=\mathrm{H}, \mathrm{R}_{4}=\mathrm{H}$ 116: $\mathrm{R}_{1}=\mathrm{CH}_{3}, \mathrm{R}_{2}=\mathrm{CH}_{3}, \mathrm{R}_{3}=\mathrm{CH}_{3}, \mathrm{R}_{4}=\mathrm{H}$ 117: $\mathrm{R}_{1}=\mathrm{CH}_{3}, \mathrm{R}_{2}=\mathrm{H}, \mathrm{R}_{3}=\mathrm{CH}_{3}, \mathrm{R}_{4}=\mathrm{H}$ 118: $\mathrm{R}_{1}=\mathrm{CH}_{3}, \mathrm{R}_{2}=\mathrm{CH}_{3}, \mathrm{R}_{3}=\mathrm{CH}_{3}, \mathrm{R}_{4}=\mathrm{CH}_{3}$ 123: $\mathrm{R}_{1}=\mathrm{H}_{1} \mathrm{R}_{2}=\mathrm{CH}_{3} \mathrm{R}_{3}=\mathrm{H}, \mathrm{R}_{4}=\mathrm{H}$

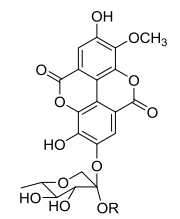

110: $\mathrm{R}=\mathrm{CH}_{3} \mathrm{CO}$ 115: $\mathrm{R}=\mathrm{H}$ 


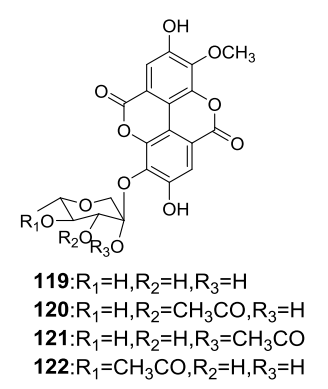

Figure 5 Chemical structures of tannins isolated from $E$. globulus.

\section{Bioactivites}

Modern pharmacological studies showed that the chemical components of E. globulus had various phamacological effects, such as antibacterial, antiviral, antitumor, antioxidant and hypo-glycemic. ${ }^{[22-24]}$

\section{Antimicrobial activity}

Tan et al. ${ }^{[7]}$ reported that compound globulol (5) had antimicrobial activity. The results revealed that the median effective inhibitory concentration $\left(\mathrm{IC}_{50}\right)$ values of globulol $(\mathbf{5})$ on Alternaria solani, Fusarium oxysporum $f$. sp. niveum, $F$. graminearum, Rhizoctonia solani and Venturia pirina were 47.1, $114.3,53.4,56.9,32.1$ and $21.8 \mu \mathrm{g} / \mathrm{mL}$, respectively. The results indicated that $\mathbf{5}$ could be a main antimicrobial compound in the ethanol crude extract of $E$. globulus.

$\mathrm{Li}$ et al. ${ }^{[25]}$ evaluated the antibacterial and antioxidant activities of $E$. globulus cultivated in Algeria. The results showed the extract exhibited moderate inhibition of lipid peroxidation of linoleic acid emulsion (51.34 $\pm 0.72 \%)$ and high reducing power $\left(\mathrm{IC}_{50}=39.52 \mu \mathrm{g} / \mathrm{mL}\right)$. It also exhibited strong antibacterial activity against $B$. subtilis and $S$. aureus with minimum inhibitory concentration (MIC) values of 30 and 80 $\mu \mathrm{g} / \mathrm{mL}$, respectively.

Various studies identified that macrocarpal A (63) and macrocarpal B (64) had strong antibacterial activity against cariogenic and periodontopathic bacteria with MIC of $0.39-1.56$ and $0.39-3.13 \mu \mathrm{g} / \mathrm{mL}$, respectively. ${ }^{[26]}$

\section{Immunosupressive activity}

Compounds 62-74 were investigated for their immune suppressive effects in vitro, and eucalyptin B (62) and macrocarpal $A(63)$ displayed moderate inhibitory activities with $\mathrm{IC}_{50}$ values of $11.8,10.2,18.2$ and $19.1 \mu \mathrm{M}$, respectively. ${ }^{[15]}$

\section{Antibacterial activity}

Li et al. ${ }^{[25]}$ reported that $E$. globulus extract was effective against the two Gram-positive strains (S. aureus, B. subtilis). The results revealed that the extract of eucalypt and gallic acid exert bactericidal effects against $S$. aureus $(\mathrm{MBC} / \mathrm{MIC}=1$ and 1.4, respectively), while tannic acid exerts a bacteriostatic effect on the same bacterium (MBC/MIC $\approx 3.33$ ). The extract and tannic acid had a bacteriostatic effect against $B$. subtilis (MBC/MIC $\approx 13.33$ and 40 , respectively), whereas gallic acid exhibited a bactericidal effect against this bacteria (MBC/MIC $\approx$ 1.33).

\section{Antitumor activity}

Migration and invasion of cancer cells into surrounding tissue and vasculature is an important initial step in cancer metastasis. Metastasis is the leading cause of cancer related death. ${ }^{[27]}$ Yang et al. ${ }^{[13]}$ reported that eucalyptin $A(46)$, together with two known compounds macrocarpal $A$ (63) and macrocarpal B (64) exhibiting potent inhibition on HGF/c-Met axis. Compound 46 showed potent inhibition on HGF-induced
c-Met activation and further suppressed HGF-stimulated cell motility and invasive behaviors.

Pham et al. ${ }^{[28]}$ evaluated the cytotoxic activity of compound eucalyptin B (62), which exhibited potent cytotoxicity against A549 cells with an $\mathrm{IC}_{50}$ value of $1.51 \mu \mathrm{M}$ and induced concentration dependent apoptosis of up to $49 \%$. Compounds euglobal-V (70) and euglobal-III (71) exhibited moderate cytotoxic effects in A549 (29.9 and $13.8 \mu \mathrm{M})$, in 4T1 (18.77 and $8.45 \mu \mathrm{M})$, and in B16F10 (8.86 and $29.33 \mu \mathrm{M})$, respectively. However, macrocarpal $C(65)$ displayed significant cytotoxicity in all three A549, 4T1 and B16F10 cells with $I_{50}$ values of 4.59 , $1.54,3.73 \mu \mathrm{M}$, respectively.

Wang et al. ${ }^{[12]}$ reported that two new phloroglucinols, eucalyptals $D(44)$ and $E(45)$, along with a related known compound, were isolated from E. globulus and their cytotoxic activity were evaluated. The results revealed that compounds 49, 50, 52 exhibited significant in vitro cytotoxicities against a few human cancer cell lines (Huh-7, Jurkat, BGC-823 and KE-97) using the CellTiter-Glo ${ }^{\mathrm{TM}}$ luminescent cell viability assay method.

Qin et al. ${ }^{[14]}$ reported that eucalypglobulusal F (54) exhibited cytotoxicity against the human acute lymphoblastic cell line (CCRF-CEM) with an $\mathrm{IC}_{50}$ value of $3.3 \mu \mathrm{M}$.

Taken together, these compounds showed significant in vitro cytotoxicities against a few human cancer cell lines. The obtained findings may lead to the development of new anticancer drugs.

\section{Other activities}

Qin et al. ${ }^{[14]}$ reported that eucalypglobulusal A (49), macrocarpal A (63), macrocarpal B (64), and macrocarpal D (66) exhibited DNA topoisomerase I (Top1) inhibition.

Ikawati et al. ${ }^{[29]}$ found that the extract of E. globulus had antihistamine activity. The results of screening indicated that five alcoholic extracts and two hexane extracts inhibited $\mathrm{lgE}$-dependent histamine release from RBL-2H3 cells.

\section{Conclusions and Perspectives}

In conclusion, the plant from the E. globulus has potent medicinal values. In recent years, many compounds have been obtained from E. globulus. Modern pharmacological studies showed that the chemical components of $E$. globulus have various pharmacological effects such as antibacterial, antiviral, antitumor, antioxidant and hypoglycemic. At the same time, the biological activities of $E$. globulus have potential research prospects. Some new chemical constituents and bioactivities are being observed, which will offer better medicinal values for clinical application in future. However, in-depth investigations on the mechanism of action are rarely reported in modern researches. For more rationally and effectively to develop resources in $E$. globulus, researchers should be strengthened on this aspect.

\section{Acknowledgement}

This work was supported by the Science and Technology Tackling Project of Henan Science Technology Department (No. 182102310063), the Key Scientific Research Project in University of Henan Province (No. 17A350013), and the National Natural Science Foundation of China (No. U1904136)

\section{Conflict of Interest}

The authors declare no conflict of interest.

Copyright (O 2021 Shudan Zheng, Hui Fang, and Jifeng Liu. This article is an open access article distributed under the terms and conditions of the Creative Commons Attribution (CC BY) license 
(http://creativecommons.org/licenses/by/4.0/). The use, distribution or reproduction in other forums is permitted, provided the original author(s) or licensor are credited and that the original publication in this journal is cited, in accordance with accepted academic practice. No use, distribution or reproduction is permitted which does not comply with these terms.

\section{References}

[1] Zhang, G. J.; Yang, Y. Y.; Zhang, S. Y.; Xu, Y. J.; Liu, T. H. Research on chemical composition of Eucalyptus globulus. Western. J. Trad. Chin. Med. 2014, 27, 162-165.

[2] Chen, B.; Zhu, M.; Xing, W. X.; Yang, G. J.; Mi, H. M.; Wu, Y. T. Chemical constituents of Eucalyptus globulus. Acad. J. Second. Mil. Med. Univ. 2002, 27, 49-50.

[3] Wang, J.; Xu, J. J.; Qiao, W.; Tang, S. A. Chemical constituents from the fruits of Eucalyptus globulus. Chin. Tradit. Herbal. Drugs. 2016, 47, 4336-4339.

[4] Xiao, S. C.; Wen, M. J.; Wu, Y. Z.; Ren, W. J.; Chen, P. Q. Botanical identification of 'Yi-Kou-Zhong' (a Chinese herb drug) and its chemical constituents of essential oil. Nat. Prod. Res. Dev. 1990, 12, 51-54.

[5] Wang, J. M.S. Dissertation, Studies on the Chemical and Bioactive Constituents from the Fruits of Eucalyptus globulus. East China Normal University, Shanghai, China, 2012.

[6] Tan, M. L.; Zhou, L. G.; Huang, Y. F.; Wang, Y.; Hao, X. J.; Wang, J. G. Antimicrobial activity of globulol isolated from the fruits of Eucalyptus globulus Labill. Nat. Prod. Res. 2008, 22, 569-575.

[7] Tan, M. L.; Wang, Y.; Zhou, L. G.; Jiang, W. B. Pentacyclic triterpenes from the fruits of Eucalyptus globulus Labill. Nat. Prod. Res. 2007, 19, 232-324.

[8] Yang, X. W.; Guo, Q. M. Chemical constituents of Eucalyptus globulus. Chin. J. Chin. Mater. Med. 2007, 32, 496-500.

[9] Tian, L. W.; Zhang, Y. J.; Qu, C.; Wang, Y. F.; Yang, C. R. Phloroglucinol glycosides from the fresh fruits of Eucalyptus Maideni. J. Nat. Prod. 2010, 73, 160-163.

[10] Tian, L. W.; Xu, M.; Li, Y.; Li, X. Y.; Wang, D.; Zhu, H. T.; Yang, C. R.; Zhang, Y. J. Phenolic compounds from the branches of Eucalyptus Maideni. Chem. Biod. 2012, 9, 123-130.

[11] Sheng, Y.; Xue, J. J.; Fan, C. Q.; Miao, Z. H.; Jian, D.; Yue, J. M. Eucalyptals A-C with a new skeleton isolated from Eucalyptus globulus. Org. Lett. 2007, 26, 5549-5552.

[12] Wang, J.; Zhai, W. Z.; Zou, Y.; Zhu, J. J.; Xiong, J.; Zhao, Y.;Yang, G. X.; Fan, H.; Hamana, M. T.; Xia, G.; Hu, J. F. Eucalyptals D and $\mathrm{E}$, new cytotoxic phloroglucinols from the fruits of Eucalyptus globulus and assignment of absolute configuration. Tetrahedron Lett. 2012, 53, 2654-2658.

[13] Yang, S. P.; Zhang, X. W.; Ai, J.; Gan, L. S.; Xu, J. B.; Wang, Y.; Su, Z. S.; Wang, L.; Ding, J.; Geng, M. Y.; Yue, J. M. Potent Hgf/c-met axis inhibitors from Eucalyptus globulus: the coupling of phloroglucinol and sesquiterpenoid is essential for the activity. $J$. Med. Chem. 2012, 55, 8183-8187.

[14] Qin, X. J.; Jin, L. Y.; Yu, Q.; Liu, H.; Khan, A.; Yan, H.; Hao, X. J.; An, L. K.; Liu, H. Y. Eucalypglobulusals A-J, Formyl-phloroglucinol -terpene meroterpenoids from Eucalyptus globulus fruits. J. Nat. Prod. 2018, 81, 2638-2646.

[15] Pham, T. A.; Hu, X. L.; Huang, X. J.; Ma, M. X.; Feng, J. H.; Li, J. Y.;
Hou, J. Q.; Zhang, P. L.; Nguyen, M. T.; Xiong, F.; Fan, C. L.; Zhang, X. Q.; Ye, W. C.; Wang, H. Phloroglucinols with immunosuppressive activities from the fruits of Eucalyptus globulus. J. Nat. Prod. 2019, 82, 859-869.

[16] Tan, M. L.; Wang, Ye.; Zhou, L. G.; Jian, W. B. Lipsoluble constituents in Eucalyptus globulus Labill fruits. J. North-west Botany 2006, 20, 2146-2149.

[17] Pham, T. A.; Li, J. Y.; Zhang, B. B.; Wang, H. Chemical constituents from the n-butanol portions from the fruits of Eucalyptus globulus. J. Chin. Pharm. Univ. 2018, 49, 422-426.

[18] Yun, B. S.; Lee, I. K.; Kim, J. P.; Chung, S. H.; Shim, G. S.; Yoo, L. D. Lipid peroxidation inhibitory activity of some constituents isolated from the stem bark of Eucalyptus globulus. Med. Chem. Nat. Prod. 2000, 23, 147-150.

[19] Liu, Y. M. Ph.D. Dissertation, Studies on Chemical Constituents from the Fruits of Eucalyptus globulus and Its Quality Control. The Second Military Medical University, Shanghai, China, 2004.

[20] Sui, X. L. M.S. Dissertation, Studies on Chemical Constituents from the Fruits of Eucalyptus globulus Labill, Shandong University, Jinan, China, 2011.

[21] Guo, Q. M.; Yang, X. W. A new ellagic acid derivative from the fruits of Eucalyptus globulus Labill. Pharmazie 2005, 60, 708-710.

[22] Fan, H.; Chen, W. R. Total polyphenols extraction from the fruits of Eucalyptus globulus Labill. and content determination by folin-ciocalteu method. Guangdong Chem. Ind. 2016, 43, 30-31.

[23] Li, W. Research advances on phloroglucinol derivatives in plants of Eucalyptus L'Héritier. Chin. Tradit. Herbal. Drugs 2015, 46, 3592-3604.

[24] Tang, Y.; Li, W. Research advances on chemical constituents of Eucalyptus globulus and their pharmacological activities. Chin. Tradit. Herbal. Drugs 2015, 46, 923-931.

[25] Boulekbache-Makhlouf, L.; Slimani, S.; Madani, K. Total phenolic content, antioxidant and antibacterial activities from the fruits of Eucalyptus globulus cultivated in Algeria. Ind. Crops. Prod. 2013, $41,85-89$.

[26] Yang, X. W.; Guo, Q. M.; Wang, Y.; Xu, W.; Tian, L.; Tian, X. J. Intestinal permeability of antivirus constituents from the fruits of Eucalyptus globulus Labill. in Caco-2 Cell Model. Bioorg. Med. Chem. Lett. 2007, 17, 1107-1111.

[27] Duff, D.; Long, A. Roles for RACK1 in cancer cell migration and invasion. Cell. Signalling 2017, 35, 250-255.

[28] Pham, T.; Mohammad, I. S.; Vu, V. T.; Hu, X. L.; Birendra, C.; Ulah, A.; Guo, C.; Lv, X. Y.; Ye, W. C.; Wang, H. Phloroglucinol derivatives from the fruits of Eucalyptus globulus and their cytotoxic activities. Chem. Biod. 2018, 15, 1-9.

[29] Ikawati, Z.; Wahyuono, S.; Maeyama, K. Screening of several Indonesian medicinal plants for their inhibitory effect on histamine release from RBL-2H3 cells. J. Ethnopharmacol. 2001, 75, 249-256.

[30] Osawa, K.; Yasuda, H.; Morita, H.; Takeya, K.; Itokawa, H. Macrocarpals $\mathrm{H}, \mathrm{I}$, and $\mathrm{J}$ from the leaves of Eucalyptus globulus. $\mathrm{J}$. Nat. Prod. 1996, 59, 823-827. 